REPORT \# RU93-5-B

\title{
DEFORMATION OF CFT BEYOND THE FIRST ORDER AND CLOSED STRING FIELD THEORY ウ̈
}

\author{
Gregory Pelts \\ Department of Physics \\ The Rockefeller University \\ 1230 York Avenue \\ New York, NY 10021-6399
}

\begin{abstract}
A self-consistent string field theory with interaction is formulated. The symmetry algebra of this theory includes, in the low-energy limit, local space-time symmetries, and the Brans-Dicke equation describes a class of low-energy solutions.
\end{abstract}

*Talk given at the conference Journées Relativistes'93 held on April 5-7 1993 at l'Université Libre de Bruxelles. To be published in the International Journal of Modern Physics D.

${ }^{\dagger}$ Supported by the DOE grant DOE-91ER4651 Task B 


\section{Introduction}

The situation in the string theory is inverse to that in all other theories. The Feynman rules, also called Polyakov rules, are known, and physicists are trying to restore from them a classical theory and symmetries. This is important for decoupling of nonphysical states, understanding the nonperturbative structure and establishing connection with space-time geometry. There is a belief that classical closed string states can be associated with quantum conformal field theories in two dimensions (CFT), which are usually defined as theories of the single string moving in some nontrivial space-time background. The condition of anomaly cancellation leads to the so-called $\beta$-function equation on the background fields. The main advantage of this approach is its more or less explicit connection to space-time geometry and the main drawback that it usually focuses only on massless fields. Treatment of massive fields is problematic, and, therefore, characterization of dynamical degrees of freedom is obscure.

Alternative approaches [1, 2, 3, 4, 5] are based on the operator formalism [6]. In [1, 2, 3] a direct connection has been found between (1,1)-primary fields of arbitrary mass level and deformations of CFT. Here, it will be shown how to generalize this result for treating of finite deformations and to formulate the string field theory.

\section{Axiomatic Conformal Field Theory}

The operator definition CFT [6] can be reformulated as the following set of axioms:

Axiom 1 There is defined a functor $\mathcal{H}$ from the category of oriented closed contours to the category of Hilbert spaces.

It means that to any oriented contour $\Gamma$ there corresponds a Hilbert space

$\mathcal{H}^{\Gamma}$ and to any even map $\Gamma_{1} \stackrel{\nu}{\longrightarrow} \Gamma_{2}$ there corresponds an isomorphism of the Hilbert spaces $\mathcal{H}^{\Gamma_{1}} \stackrel{\hat{\nu}}{\longrightarrow} \mathcal{H}^{\Gamma_{2}}$ in such a way that $\hat{\nu_{1}} \widehat{\circ} \nu_{2}=\hat{\nu_{1}} \hat{\nu_{2}}$. To a multicomponent contour $\Gamma=\bigcup_{j} \Gamma_{j}$ we will assign the space $\mathcal{H}^{\Gamma}=\bigotimes_{j} \mathcal{H}^{\Gamma_{j}}$.

Axiom 2 The spaces corresponding to the contrary-oriented contours are conjugated. 
Axiom 3 To each bordered Riemann surface $\Sigma$ there corresponds in a conformal invariant way a specific element $\mathcal{A}_{\Sigma}$ of $\mathcal{H}^{\partial \Sigma}$ (amplitude) such that

$$
\mathcal{A}_{\Sigma}=\operatorname{Sp}\left(\mathcal{A}_{\Sigma_{\Gamma}}\right) .
$$

Here $\Sigma_{\Gamma}$ is a surface or set of surfaces resulting from the cutting of $\Sigma$ along closed contour $\Gamma$, and $\mathrm{Sp}$ is an operator contracting components of $\mathcal{H}^{\partial \Sigma_{\Gamma}}$ corresponding to two contrary oriented copies of $\Gamma$.

For the surface with the one and two component boundary, $\mathcal{A}_{\Sigma}$ can be considered to be the vacuum state and the propagator, respectively. To a multicomponent surface $\Sigma=\bigcup_{j} \Sigma_{j}$ we will assign the amplitude $\mathcal{A}_{\Sigma}=\bigotimes_{j} \mathcal{A}_{\Sigma_{j}}$. In order to describe CFT with central charge we should relax the axioms above, changing them to their projective analogues.

\subsection{Vertex operators}

We will say that an element $\Psi$ of $\mathcal{H}^{\partial \Sigma}$ has support in point $z_{0} \in \Sigma$, if for any contour $\Gamma$ surrounding $z_{0}$ counter clockwise it can be written as $\Psi=\mathcal{A}_{\Sigma_{\text {ext }}} \Psi_{0}$, where $\Psi_{0} \in \mathcal{H}^{\Gamma}$ and $\Sigma_{\text {ext }}$ is an external component of $\Sigma_{\Gamma}$. Considering the map $\Psi \longrightarrow \Psi_{0}$ as an equivalence relation, we can identify the spaces of states corresponding to different areas of the Riemann surface but having the same support. The Virasoro algebra can be shown to have a natural representation in the space $H_{z_{0}}$ resulting from such identification.

The space $H_{z_{1}, \ldots, z_{N}}$ of states having support in a set of points $z_{i}$ is equivalent to $\bigotimes_{i=1}^{N} H_{z_{i}}$. Elements of $H_{z_{i}}$ and the map $H_{z_{1}} \times \ldots \times H_{z_{N}} \longrightarrow H_{z_{1}, \ldots, z_{N}}$ and can be interpreted as vertex operators and their $T$-product.

\section{Deformation of CFT}

Let $\Psi$ be some vertex operator field (not necessarily primary). If for deformed amplitude we will use the formula

$$
\mathcal{A}_{\Sigma}^{\prime}=\operatorname{Texp} \frac{1}{\pi} \int_{\Sigma} \Psi d^{2} z,
$$

the condition (2.1) will be automatically satisfied and only the condition of conformal invariance will remain to be implemented. 


\subsection{Regularization}

We can not use the simple cutoff regularization

$$
\int_{\Sigma} \phi d^{2} z \longrightarrow \int_{\left\{z \in \Sigma,\left|z-z_{j}\right| \geq r_{j}\right\}} \phi d^{2} z
$$

for integration of point-like contact singularities in (3.1) because it will violate the condition (2.1). Instead, we should use an average of such regularization over cutoff parameters $r_{j}$ with the generalized measure $\mu$ in $\mathbf{R}_{+}$defined by the formula

$$
\int_{0}^{\infty} r^{2 \alpha} \mu(r) d r=\Lambda(\alpha) \quad(\alpha \in \mathbf{R})
$$

Here, $\Lambda$ is some smooth function on $\mathbf{R}$ satisfying $\Lambda(0)=1$ and $\Lambda(\alpha)=0$ for $\alpha$ bigger then some positive number. A related proposal for regularization corresponding to specific stepfunction $\Lambda=\Theta(-\alpha) d^{\alpha}$ was independently made in [5]. It can be shown that the way in which the boundary singularity is regularized is not important as it does not affect the equivalence class of CFT.

\section{Equation of Motion}

Let us identify deformed CFT vertex operators with initial CFT vertex operators by means of the formula

$$
\Xi_{\Psi}=T\left(\Xi \exp \left(\frac{1}{\pi} \int \Psi d^{2} z\right)\right)
$$

Deformed right and left components of energy momentum tensor can be shown to be

$$
\mathcal{T}^{\prime}=(\mathcal{T}+\Phi)_{\Psi}, \quad \overline{\mathcal{T}}^{\prime}=(\overline{\mathcal{T}}+\bar{\Phi})_{\Psi},
$$

where $\Phi, \bar{\Phi}$ are some normalizable vertex operator functions. The condition

$$
\bar{d} \mathcal{T}^{\prime}=d \overline{\mathcal{T}}^{\prime}=0
$$

leads to dynamic equation on fields $\Psi, \Phi, \bar{\Phi}$ which can be interpreted as an equation of motion. In fact, it is a conformal invariance condition for the deformed CFT. 


\subsection{Symmetries}

It can be shown that the following transformations

$$
\begin{aligned}
& \delta \Psi(z, \bar{z})=\bar{d} \xi(z, \bar{z})+\operatorname{Res}_{u=z} T(\xi(u, \bar{u}), \Psi(z, \bar{z}))+\ldots \\
& \delta \Phi(z, \bar{z})=\operatorname{Res}_{u=z} T(\xi(u, \bar{u}), \mathcal{T}(z)+\Phi(z, \bar{z}))+\ldots \\
& \delta \bar{\Phi}(z, \bar{z})=\operatorname{Res}_{u=z} T(\xi(u, \bar{u}), \bar{\Phi}(z, \bar{z}))+\ldots
\end{aligned}
$$

and their conjugates do not effect the equivalency class of deformed CFT. Here, we use the residue generalized for nonholomorphic functions by the formula

$$
\operatorname{Res}_{u=z}(u-z)^{k}|u-z|^{2 \alpha}=\Lambda(\alpha) \delta_{k+1,0} \quad(\alpha \in \mathbf{R}, k \in \mathbf{Z}) .
$$

where $\Lambda$ is the function used in regularization (3.2).

We will impose on the fields a translation invariance condition.

$$
\left(d+L_{-1}\right) \Xi=\left(\bar{d}+\bar{L}_{-1}\right) \Xi=0, \quad \Xi=\Psi, \Phi, \bar{\Phi} .
$$

As regularization used is translationally invariant, it will not reduce the phys-

ical degrees of freedom. The remaining symmetries correspond to translation invariant fields $\xi, \bar{\xi}$.

\subsection{Linearization}

The linearized equation of motion (4.2) and symmetries (4.3) can be shown respectively to be

$$
L_{1} \mathcal{O}_{0} \Psi=\bar{L}_{-1} \Phi, \quad \bar{L}_{1} \overline{\mathcal{O}}_{0} \Psi=L_{-1} \Phi
$$

and

$$
\delta \Psi=L_{-1} \bar{\xi}+\bar{L}_{-1} \xi, \quad \delta \Phi=L_{1} \mathcal{O}_{0} \xi, \quad \delta \bar{\Phi}=\bar{L}_{1} \overline{\mathcal{O}}_{0} \bar{\xi} .
$$

Here and afterwards

$$
\mathcal{O}_{k}=\delta_{k, 0}+\sum_{j=0}^{\infty} \frac{\left(L_{-1}\right)^{j} L_{k+j}}{(k+j+1) !}, \quad \overline{\mathcal{O}}_{k}=\delta_{k, 0}+\sum_{j=0}^{\infty} \frac{\left(\bar{L}_{-1}\right)^{j} \bar{L}_{k+j}}{(k+j+1) !} .
$$

The equation (4.4) is a relaxation of the conventional closed string equation

$$
\Phi=0, \forall k \geq 0:\left(L_{k}+\delta_{k, 1}\right) \Psi=\left(\bar{L}_{k}+\delta_{k, 0}\right) \Psi=0 .
$$


This relaxation is compensated by the symmetries (4.5) and does not create additional physical degrees of freedom. The deformed representation of the Virasoro algebra in the linear approximation can be shown as follows

$$
\begin{gathered}
L_{k}^{\prime}-L_{k}=\operatorname{Res}_{z=z_{0}}\left(z-z_{0}\right)^{k+1} \Phi(z, \bar{z})+\operatorname{Res}_{\bar{z}=\bar{z}_{0}}\left(z-z_{0}\right)^{k+1} \Psi(z, \bar{z})- \\
\operatorname{Res}_{\bar{z}=\bar{z}_{0}} \sum_{j=0}^{k+1} \frac{(k+1) !}{j !}\left(z-z_{0}\right)^{j} \mathcal{O}_{k+1-j} .
\end{gathered}
$$

In the case of the primary field solution (4.6), only the second term in (4.7) contributes and gives a regularized version of the deformation suggested in [[1, 2]. Calculations made up to the third order have shown that in the lowenergy limit the theory includes local space time symmetries and a class of solutions described by the Brans-Dicke equation. It generalizes the analogous result [7, 8] found in the linear approximation and gives one more proof of the self-consistency of the presented formalism.

\section{Conclusion}

The theory presented here can be considered to be a closed string field theory, because to its solutions there explicitly correspond CFT and, in the first approximation, states of the free closed string. Besides, the low-energy limit can be related to Brans-Dicke theory.

\section{Acknowledgements}

Author thanks Mark Evans for inspiring him to develop the deformation approach, for discussions and for many important suggestions.

\section{References}

[1] M. Evans and B. Ovrut, Deformations of Conformal Field Theories and Symmetries of the String, Phys. Rev. D41 (1990) 3149

[2] A. Sen, On the background independence of string field theory, Nucl. Phys. B345 (1990) 551 
[3] M. Campbell, P. Nelson and E. Wong, Stress tensor perturbations in conformal field theory, Int. J. Mod. Phys. A6 (1991) 4909

[4] B. Zwiebach, Closed string field theory: quantum action and the BatalinVilkovisky master equation, Nucl. Phys. B390 (1993) 33, hep-th/9206084

[5] K. Ranganathan, H. Sonoda and B. Zwiebach, Connections on the statespace over conformal field theories, preprint FERMILAB-PUB-MITCTP-2193, April 1993, hep-th/9304053

[6] Alvarez-Gaume, C. Gomez, G. Moore and C.Vafa, Strings in the operator formalism, Nucl. Phys B303 (1988)445

[7] M. Evans and I. Giannakis, Gauge-covariant deformations, symmetries, and free parameters of string theory, Phys. Rev. D44 (1991) 2467

[8] M. Evans and I. Giannakis, Deformations, symmetries and topological degrees of freedom of the string, in Proceedings of the XX International Conference on Differential Geometric Methods in Theoretical Physics, June 3-7, 1991 (S. Catto and A. Rocha, eds.), World Scientific (Singapore), p. 759, hep-th/9109055 